\title{
The basic cycle of reorganization of construction
}

\author{
Yegor Kotov* and Vitaly Chulkov \\ Moscow State University of Civil Engineering, Yaroslavskoe shosse, 26, Moscow, 129337, Russia
}

\begin{abstract}
Reorganization is one of the fundamental processes of development of modern society. The infographic model of the reorganization cycle, also known as the "development wheel", allows us to divide development of this process into four stages: settlement, disorganization, reorganization and co-organization. These stages have been researched on by numerous Russian and foreign researchers. In the sphere of construction, the most interesting is the restructuring, which has a special relevance at the present stage of development of the Russian Federation and requires creative approaches, models and methods of scientific research of vision of this important branch of economy. In this article, the model of reorganization process is structured with use of the theory of multipoint logic and the practice of infographic modeling, possible impacts and interactions leading to well-defined and formalized results are considered. Models of loading the interactions of individual components of the model of reorganization by control actions and the results obtained therewith have been developed.
\end{abstract}

\section{Introduction}

An objectively existing continuous process, in certain periods of which, by desire or by necessity, it is possible to artificially maintain the stability of a limited number of system parameters in limited ranges of its operation is called reorganization. This is a way to transform physical, virtual, legal, and other objects and processes of activity (their merger, separation, accession, termination, emergence, etc.), allowing the possibility of partial or fundamental change in organizational, legal forms, technologies, methods and means of functioning of objects of reorganization. During reorganization, there are used both existing and newly-proposed, or already existing in any other objects organizational-legal, organizational-technological, and legal forms, methods and means.

The analysis of the problems of reorganization (as a fundamental process of developing activities in key sectors of economy) and enhance of the effectiveness of processes of construction industry has been made by numerous foreign and Russian researchers and scientists (M. Povey, T. Lloyd-Jones [1], N. Kohler, T. Lützkendorf [2, 3], M. Lu, H. Li [4], MP. Nepal, M. Park [5], H. Millward [6], A. Duffy [7], L. Kamal-Chaoui, E. Levan, Z. Fufei [8], A. Deborah, T. Abowitz ,T. Mihael [9], S. Salat, L. Bourdic, C. Nowacki

\footnotetext{
*Corresponding author: egor.kotov.95@bk.com
} 
[10,11], C. Weible, D. Main, T. Heikkila, S. Siddiki, A. Duvall, A. Pattison, M. Bernard [12], J. Jaeger, C. Schwick [13], Qing Fan, Hongqin Fan [14], Yu. Monfred, B. Prykin [15], A. Gusakov[16], A. Schegol [17], V. Karpenkov[18], V. Chulkov [19-21], A. Semechkin [22], B. Prykin [23], N. Golubeva [24], A. Prigogine [25], and others).

For most types of human activity reorganization is a constantly used specific sequence of organizational and technological operations or processes. This sequence is displayed either as a closed loop (reorganization cycle), or as an open loop (reorganization network).

The cycle (greek kyklus - circle) in infographic modeling [19-22] is a closed circuit (a sequence of individual events of activity). The direction of movement of the cycle (the sequence of the implementation of cycle events clockwise or against it) is chosen depending on the formulation of the problem to be solved. Any of the events of the cycle can be chosen as its starting (at the same time ending) point. A complete cycle is a cycle that sequentially implements all the events included in it. In the absence of implementation of at least one of the events forming it, the cycle is called illuminated. Illumination is the implementation of exclusively necessary cycle events for solving a specific task.

The reorganization network (open loop of the reorganization cycle) is referred to as composite infographic scheduling models that allow: displaying the dynamics of changes in the intensity indicators of activity processes over time; understand what activities should take place according to plan, and what actually takes place; reduce or increase the duration of implementation of events of the cycle.

The study of reorganization, as one of the important fundamental processes of development of modern society, includes: modeling of parameters, properties, impacts and interactions of individual components of the cycle and reorganization network; identification (planning and design) of the expected and actual results of fixed interactions in the cycle and reorganization network.

\section{Methods}

The main method of scientific research was chosen [19-22] visual-figurative infographic modeling of the fundamental reorganization process as a whole, its individual components and their interactions. The infographic model of the basic cycle of reorganization is shown in the figure 1. It is a sequence of four stages (phases): Ar - "arrangement"; Di "disorganization"; Re - "reorganization"; Co - "co-organization". This model reveals two previously comprehensively unexamined relationships: arrangement-reorganization (Ar↔Re); co-organization-disorganization (Co↔Di). 


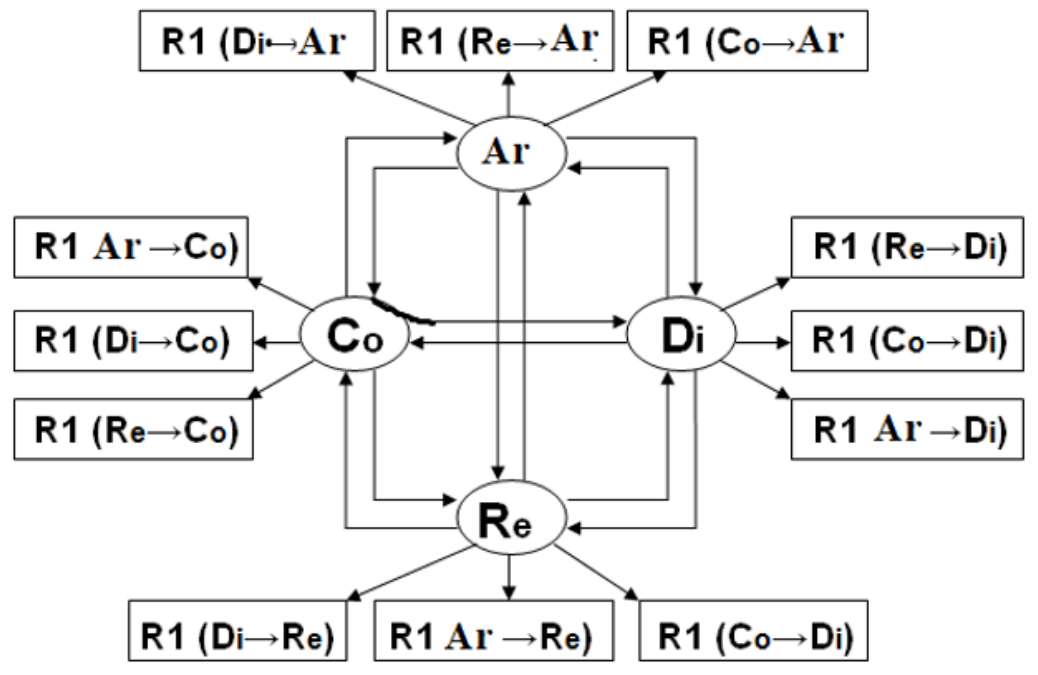

Fig. 1. Results of R1 impacts of the components of the reorganization cycle (V. Chulkov, 2017)

Solid one-sided arrows of exposure between stages Ar, Di, Re and Co correspond to the idealized quasi-static process of transition from the previous stage of the cycle to the next one (B. Prykin calls this process quasi-equilibrium [23]). Quasi (lat. quasi: something like, as if, as it were) - a prefix that forms nouns with the meaning of falsity, imaginary (quasiequilibrium, quasi-stability, etc.).

Transforming the opposite stages (Ar and Di, Re and Co) into each other is a quasistatic reversible process consisting of continuously following unstable equilibrium states in conditions of constant entropy. At the same time, the transformation of the De $\leftrightarrow$ Re allows one to estimate the state of the object being reorganized, while Co $\leftrightarrow \mathrm{Di}$ allows one to estimate the state of the processes of reorganization of this object.

The value $\mathrm{V}$ of the reorganization network (according to $\mathrm{R}$. Metcalf) increases as its components grow in proportion to the square of the number of these components $\mathbf{n}^{2}$ [23]:

$$
V=(n-1) \times c
$$

where: $c=$ const is the assessment of the possibility of interaction with one of the network components (the implementation of this component). The integral value of the reorganization network $P n$ of $n$ components (events) is determined by the expression

$$
P n=n \times(n-1) \times s
$$

The value of $P n$ increases according to the quadratic law. The value of connecting to a network (participating in some reorganization process) for a particular participant in the reorganization is the higher, the greater the number of participants are involved in this process ("network effect"). The concept of "network effect" is also known by other names, for example: network externalities; demand-side economies of scale; positive feedback.

Let us consider in more detail the content of the performed four stages (phases "arrangement", "disorganization", "reorganization" and "co-organization") of the infographic model of the basic reorganization cycle.

Arrangement (order, stable operation) - a technical, social or organizational object designed to perform certain functions without limiting the resources used to achieve the result. The "arrangement" (ordering, functioning according to the rules) is necessary in order to reduce the diversity of elements and states of the environment in the "man-technics 
-environment, MTE" system, to prevent the collision of many interests, an infinite number of which creates a conflict environment where it is difficult and dangerous not only to function, but just to be.

Disorganization (french. Désorganisation - violation of order, disorder) - the destruction of the existing order, setting discord in coordinated actions, analysis of vulnerable points and conflicting organizational situations at all levels, the centrifugal process with a destructive tendency. "Disorganization" is defined as one of the options for the interaction of three realities: natural (natural phenomena and the consequences of their interaction); an artificial set of models of peoples mental activity; physical implementations of these models. When disorganization, on the background of a decrease in the organizational and managerial level, the system eliminates the superfluous and unnecessary. Disorganization is always the destruction of an existing order, operating norms of functioning. This "destruction under the auspices of rule-making" is not only the performers, but also the creators of the rules, intentionally or deliberately doing this. In disorganization, there are several stages [25]: loosening of the "arrangement" (the boundaries of the rules become uncertain, their implementation or deviation from them is difficult to estimate); non-observance of the "arrangement" (some participants of interaction follow the rules, while others do not, or follow other rules); inconsistency of the "arrangement" (some rules exclude others, the execution of one means the violation of the other); the destruction of the "arrangement" (the behavior of most people functioning in the existing "arrangement" denies this "arrangement").

Restructuring - the arrangement of something anew, according to a new plan, on new grounds to achieve new goals with new means. This is a global trend and the main process of development in all aspects of its manifestation (personal, collective, functional, professional, communication, historical, technical, etc.).

Co-organization - "tightening" of diverging or conflicting interests, creating new and restoring existing organizational forms, a mechanism for coherence, creating an orderly system, a constant objective need of any society, a collective process with a centripetal tendency. The methods and forms of co-organization determine as a result of the analysis of vulnerable points and conflicting organizational situations of all levels of disorganization: interpersonal and intergroup relations, interaction of institutions, enterprises, administrations and states. When co-organizing, new methods and technologies of interaction arise against the background of crowding out the previously ineffective "old" ones that already exist, which leads to disorganization of the system components. Coorganization and disorganization are the opposite (antonymic), but closely interrelated development processes of any open system, stages of its evolutionary or volitional reorganization.

\section{Discussion}

In construction sphere the most interesting component of reorganization cycle is restructuring that has a special relevance at the present stage of development of the Russian Federation and requires creative approaches, models and methods of scientific research on the development prospects of this important branch of economy.

In this article involving the theory of multipoint logic and the practice of infographic modeling, the model of the reorganization process is structured, possible impacts and interactions are considered, leading to well-defined and formalized results (Fig. 1).

Analysis of domestic and foreign publications allows us to generalize the ratio of "arrangement" and "reorganization" in our country by the end of 2018. as 20:80, and the trend of increasing the share of the conversion does not change. This assessment allows us 
to consider the "arrangement" as a fictitious-demonstrative product (G. Shchedrovitsky's term) of "reorganization" and at the same time a stimulus to a new stage of restructuring.

The nature of the dialectical interrelation and the predominance of one of the concepts in the "arrangement - restructuring" dyad is always resource. The moment of crisis (for example, a change in the state of "arrangement" to state of "restructuring") occurs when there are not enough resources to maintain stability, but there are enough resources for its destruction, restructuring and the achievement of another variant of "stability", that is, for organizing another fictitious-demonstration product, always presented as "finally achieved, true".

In modern forms and processes of restructuring, a significant part of which is initiated by business, it is easy to notice the fact of deliberately discrediting the concept of "serve" as a conscious disinterested participation in the constantly implemented processes of maintaining and developing the social structure, and replacing it with the concept of "service" meaning a commodity having a certain value. The paradox is that these concepts are not mutually exclusive, each having its own area of application, and the services are false. Excesses of this kind are characteristic of any poorly prepared and not thoroughly thought out quick restructuring.

The judgments listed above accentuate the subjectivity of the processes and results of, when "restructuring" is an objectively existing manifestation of development in society. This is a continuous process, at certain stages and at certain intervals of the realization of which, if desired or necessary, it is possible to fix the stability of limited parameters in limited ranges of their realization for a limited part of the society. Such states, as mentioned above, can be called the "arrangement", the antithesis of "restructuring".

In most cases, the perception of the inevitable "restructuring" results in an attempt to strictly define it from the point of view of what had previously seemed unchanged and true; if the new does not fit into the peoples old picture of the world, they propose to fight it.

In fact, if the purpose is to seize the human consciousness, it should, without traumatizing it, help to combine the existing picture of the world of this person with the ideas of the authors of the restructuring (to carry out "co-organization").

\section{Results}

Identifying and modeling one of the most common management processes - loading the "contour" and "diagonal" interconnections of two components of the basic reorganization model (Fig. 2.) by two independent control actions not involved in this relationship can be considered a significant result of a scientific study of the reorganization process components (Fig.3.). The results of effects of $\mathbf{C o} \rightarrow \mathbf{R e}$ and $\mathbf{R e} \rightarrow \mathbf{C o}$, as well as the results of "loading" by control actions of the interconnection of $\mathbf{C o} \leftrightarrow \mathbf{R e}$ are formalized (in Fig. 4 the conventional loading symbol $\boldsymbol{\nabla}$ is used).

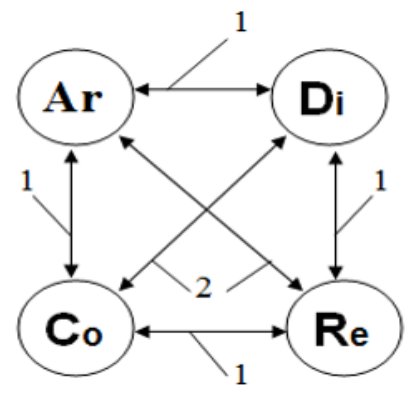

Fig.2. "Outline" (1) and "diagonal" (2) interactions of the components of the model (Fig. 1.) 

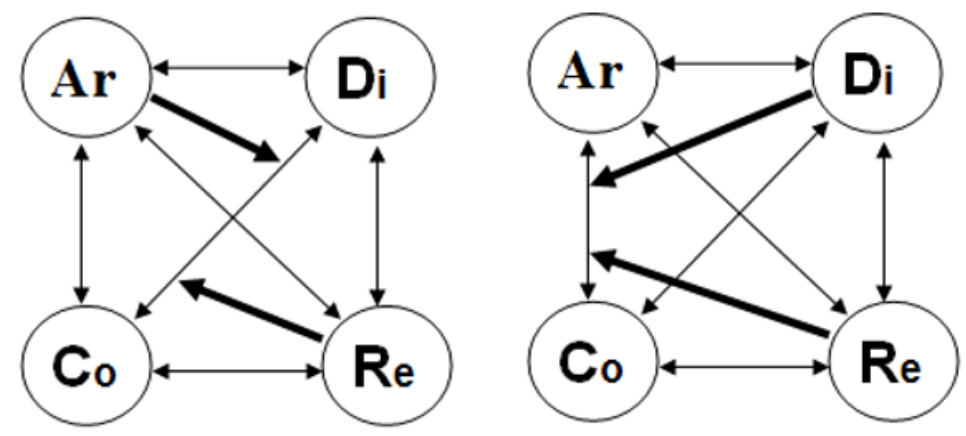

Fig.3. Loading "diagonal" (a) and "contour" (b) interconnection by control actions

The use of formal descriptions of the effects and interactions results (Fig. 1 and Fig. 4.) allows the researcher to: be guided in a variety of possible options for solving problems of reorganization management; optimize the volume of control and loading effects in the process of reorganization; implement the "digitalization" (this innovative term is now widely used by mass media) of the quality control of the reorganization within the framework of the information computer technology used.

The main methods for studying the totality of traditional and innovative indicators of reliability and efficiency of reorganization processes in construction, as well as their interrelationships, can be considered as factor and correlation and regression analysis. These methods allow to study the relationship between the parameters of variables in their individual sets or between such sets. At the same time, covariance or correlation matrices are considered, determining the similarity and difference of the values of individual parameters and ensuring the reliability of the results of scientific research. In the course of the research, the current values of the parameters are identified and measured, reasonably selected for a comprehensive analysis, determining the degree of influence of each of them on the resulting actual value of the parameter.

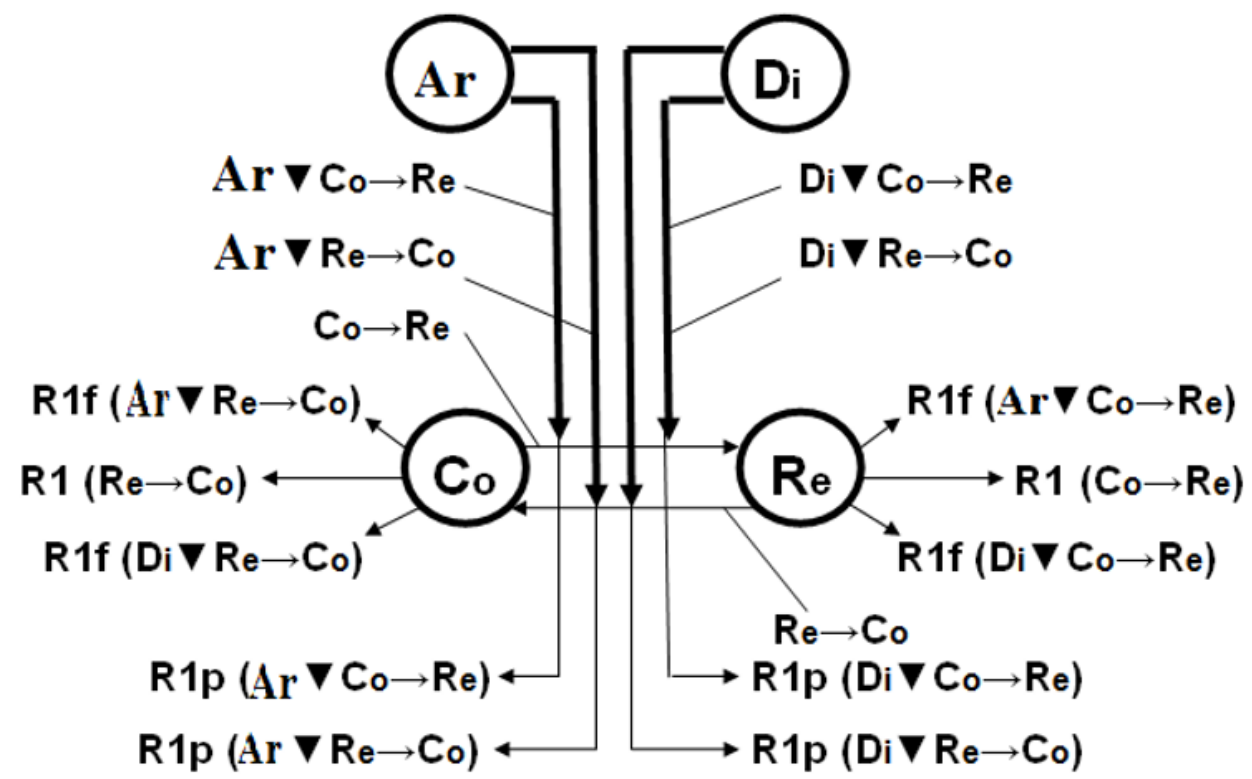

Fig.4. The results of the effects of R1 and "loading" R1p in the model (Fig. 1) 
A scientific study of reorganization in construction was carried out using the following varieties of the method of factor analysis of process reliability and efficiency. Deterministic analysis reveals the logic of the impact of the main factors of reorganization based on the quantitative values of the process parameters, and stochastic analysis is used when several other indicators change to varying degrees with changes in one factor. Direct (deductive) factor analysis leads the study from the general to the particular, and the reverse (inductive) - from the particular to the general. Single-stage factor analysis examines the factors of one level of subordination without their detailed structuring. Multistep analysis assumes the specification of reorganization factors located at different levels of the building production hierarchy with the identification of inter-level links. Static analysis examines the calendar interrelation of reorganization factors, and the dynamic examines the cause-effect relationships in their development. The reasons for the changes in the interrelations of factors in previous periods of time investigate retrospective factor analysis, and the forecast of possible changes in factors and their modeling are performed by a perspective factor analysis.

The result of a scientific study of reorganization in construction are private and comprehensive indicators of reliability and efficiency. The most generalizing of them should be considered the readiness factor of the construction industry (the ratio of the time of failure-free organizational and technological reorganization to the full period of the observation period).

Defined the average values of the ranges of variation of the availability factor for: technical means of mechanization and transportation: $0.86-0.92$; material resources and components: 0.80-0.85; labor resources: 0.78-0.83.

\section{Summary}

Using the theory of multipoint logic and the practice of infographic modeling, a model of the reorganization process is structured, four stages of this process (arrangement, disorganization, restructuring and co-organization) are revealed. The most interesting stage is the restructuring, which has a special relevance and requires new original approaches and concepts of development. A further direction of the scientific study of construction restructuring is the identification of traditional and innovative types of restructuring with the subsequent study of the typical properties and characteristics of each of them. Currently, the most relevant type of modern construction restructuring is renovation.

\section{References}

1. M. Povey, T. Lloyd-Jones, Mixed value urban development: Mechanisms for sustaining the livelihoods and social capital of the urban poor, ESF/NAERUS workshop (2000)

2. N. Kohler, T. Lutzkendorf, Integrated Life Cycle Analysis. Building Research and Information, 30, 338-348 (2002)

3. N. Kohler, S. Moffatt, Life-cycle analysis of the built environment, UNEP Industry and Environment April - September, 17-21 (2003)

4. M. Lu, H. Li, Resource-activity critical-path method for construction planning, Journal of Construction Engineering and Management, 129, 412-420 (2003) 
5. M. Nepal, M. Park, Downtime model developement for construction equipment management, Engineering, Construction and Architectural Management, 11, 412-420 (2003)

6. H. Millward, Urban Containment Strategies: A Case-Study Appraisal of Plans and Policies in Japanese, British and Canadian Cities, Land Use Policy 23, 473-485 (2006)

7. A. Duffy, Land use planning in Ireland - a life cycle energy analysis of the recent residential development in the Greater Dublin Area, The International Journal of Life Cycle Assessment, 14, 268-277 (2009)

8. L. Kamal-Chaoui, E. Leman, Z. Fufei , Urban Trends and Policy in China.- OECD Regional Development Working Papers (2009)

9. A. Deborah, T. Abowitz, T. Michael, Mixed Method Research: Fundamental Issues of Design, Validity, and Reliability in Construction Research.- Journal of Construction Engineering and Management, 136, 47-49 (2010)

10. S. Salat, L. Bourdic, C. Nowacki, Assessing urban complexity, International Journal of Sustainable Building Technology and Urban Development, 1 ,160-167 (2010)

11. S. Salat, L. Bourdic, C. Nowacki, Urban complexity, efficiency and resilience. In: Z Morvaj (ed.), Energy efficiency - A bridge to low carbon economy, InTech (online). (http://cdn.intechopen.com/pdfs-wm/31591.pdf). (2012)

12. C. Weible, D. Main, T. Heikkila, S. Siddiki, A Duvall, A. Pattison, M. Bernard, A social-ecological-infrastructural systems framework for interdisciplinary study of sustainable city systems, 16, 801-813 (2012)

13. J. Jaeger, C. Schwick, Improving the measurement of urban sprawl: Weighted Urban Proliferation (WUP) and its application to Switzerland, Ecological Indicators, 38, 294308 (2014)

14. Qing Fan, Hongqin Fan, Reliability Analysis and Failure Prediction of Construction Equipment with Time Series Models, Journal of Advanced Management Science, 3, 203-210, (2015)

15. Yu. Monfred, B. Prykin, Organization, planning and management of construction industry enterprises: A textbook for universities, M: Stroyizdat (1989)

16. A. Gusakov, Organizational and technological reliability of construction, M .: SVRArgus, (1994)

17. A. Shchegol, System-engineering design of scientific support of construction.- Author's abstract. diss. ... Doct. tech. sciences. Moscow: Publishing House of the DIA, (1996)

18. V. Karpenkov, Concepts of modern science, M.: UNITY, 402, (1997)

19. V. Chulkov, Geometric modeling in the complex documentation of engineering objects (infographics) Autor's abstract. dis. Publishing House of the DIA, 28, (1989)

20. V. Chulkov, Infographics, Academy of Sciences of the USSR, National Assembly by compl. problem "Cybernetics", (1991)

21. V. Chulkov Infographic models of cycles and their application in different engineering applications.- Sb. scientific tr. University of Knowledge Methodology. 3, 28-34 (1996)

22. A. Semechkin Infographic methods for organizing the reconstruction of residential quarters.- Author's abstract. diss. ... cand. tech. sciences. Moscow: Publishing House of the DIA, 18, (1999)

23. B. Prykin Technical and economic analysis of production: A textbook for universities.M.: UNITY, 399, (2000)

24. NN Golybeva, Automated design organization reorganization of urban areas with a change in their purpose, Abstract of diss. cand. tech. sciences, 18, (2004)

25. A. Prigojin, Disorganization: Causes, Types, Overcoming, M .: Alpina Business Books, 402,(2007) 